\title{
Myocardial motion estimation combining tissue Doppler and B-mode echocardiographic images
}

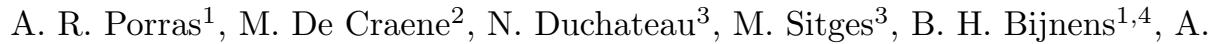 \\ F. Frangi ${ }^{1,5}$, G. Piella ${ }^{1}$ * \\ 1 Department of Information and Communication Technologies, Universitat Pompeu \\ Fabra, Barcelona, Spain. \\ 2 Philips Research, Medisys, Suresnes, France. \\ 3 Hospital Clínic - IDIBAPS - Universitat de Barcelona, Barcelona, Spain. \\ 4 ICREA, Barcelona, Spain. \\ 5 Department of Mechanical Engineering, University of Sheffield, Sheffield, UK.
}

\begin{abstract}
We present a registration framework that combines both tissue Doppler and B-mode echocardiographic sequences. The estimated spatiotemporal transform is diffeomorphic, and calculated by modeling its corresponding velocity field using continuous B-splines. A new cost function using both B-mode image voxel intensities and Doppler velocities is also proposed. Registration accuracy was evaluated on synthetic data with known ground truth. Results showed that our method allows quantifying wall motion with higher accuracy than when using a single modality. On patient data, both displacement and velocity curves were compared with the ones obtained from widely used commercial software using either B-mode images or TDI. Our method demostrated to be more robust to image noise while being independent from the beam angle.
\end{abstract}

\section{Introduction}

Ultrasound imaging (US) is one of the preferred modalities to assess myocardial motion and deformation in clinical routine, due to its non-invasiveness, costeffectiveness, and high temporal resolution (around $30 \mathrm{fps}$ for $3 \mathrm{D}$ and up to more than $100 \mathrm{fps}$ for 2D). The presence of local speckles facilitates the use of tracking algorithms to estimate motion and deformation locally, but may suffer from image quality. Complementarily, Tissue Doppler Imaging (TDI) allows the quantification of myocardial velocities with a higher temporal resolution and better signal-to-noise ratio, although with lower spatial resolution and angle dependency. However, the integration of such results remains difficult, but highly desired to overcome the disadvantages of using each technique separately.

The objective of this paper is to use both B-mode and TDI sequences to estimate a single displacement field, taking advantage of the spatial resolution

\footnotetext{
* This research was partially funded by the Spanish Ministry of Economy and Competitiveness (Ref.TIN2012-35874). The work of A. R. Porras was supported by the Spanish Government with a FPU grant.
} 
of B-mode images and the temporal resolution of TDI. In particular, we propose a new cost function including a term that measures the agreement between the estimated velocity projected on the beam direction and the velocity values obtained from TDI.

Integration of multiple US cardiac images in one single image to be used for a quantitative analysis was proposed in [1] and [2]. In addition, methods to perform an integrated analysis of different US images were also proposed in [3] and [4]. All of these methods integrate different images from the same modality. In [5], TDI was used for motion tracking along the beam direction and B-mode images were used for tracking in the transversal direction. In [6] a combined analysis of Bmode sequences and TDI was proposed, using an optical flow-based registration method with a spatial affine velocity model and a coarse-to-fine multi-scale approach. With this method, registration was performed pairwise, so the temporal coherence was not guaranteed. Moreover, B-mode and TDI frames needed to be evaluated at exactly the same time instant.

In our approach the velocity field was modeled continuously using B-spline kernels. All frames were registered simultaneously by estimating one single spatiotemporal transform to preserve both spatial and temporal consistency. To estimate this transform, the agreement between the velocity field estimated projected on the beam direction and the velocities obtained from TDI is measured, in addition to a more classical voxel intensity-based similarity metric between the registered images and a reference. In our implementation, the estimated transform is diffeomorphic, thus being smooth, invertible and with smooth inverse at every spatiotemporal location.

\section{Methodology}

The proposed algorithm is based on the Temporal Diffeomorphic Free Form Deformation (TDFFD) registration method presented in [7] and improves it by taking into account tissue velocities obtained from TDI. A summary of the proposed registration framework is illustrated in Fig. 1.

\subsection{Velocity field and transform}

The TDFFD algorithm models a continuous velocity field as a sum of spatiotemporal B-spline kernels, where the B-spline coefficents (velocities at each control point) are concatenated in a parameters vector $\mathbf{p}$ :

$$
\mathbf{v}(\mathbf{x}, t ; \mathbf{p})=\sum_{i, j, k} \beta\left(\frac{x-q^{i}}{\Delta_{i}}\right) \beta\left(\frac{y-q^{j}}{\Delta_{j}}\right) \beta\left(\frac{t-q^{k}}{\Delta_{k}}\right) \mathbf{p}^{i, j, k}
$$

where $\mathbf{x}=\{x, y\}$ are the spatial coordinates of the point whose velocity is evaluated, $\beta(\cdot)$ is a cubic B-Spline kernel function, $\mathbf{Q}=\left\{q^{i}, q^{j}, q^{k}\right\}$ represents the grid of control points and $\boldsymbol{\Delta}=\left\{\Delta_{i}, \Delta_{j}, \Delta_{k}\right\}$ is the spacing between control points. To map a point at coordinate $\mathbf{x}=\{x, y\}$ from time $t=0$ to time $t=T$, the velocity field has to be integrated: 


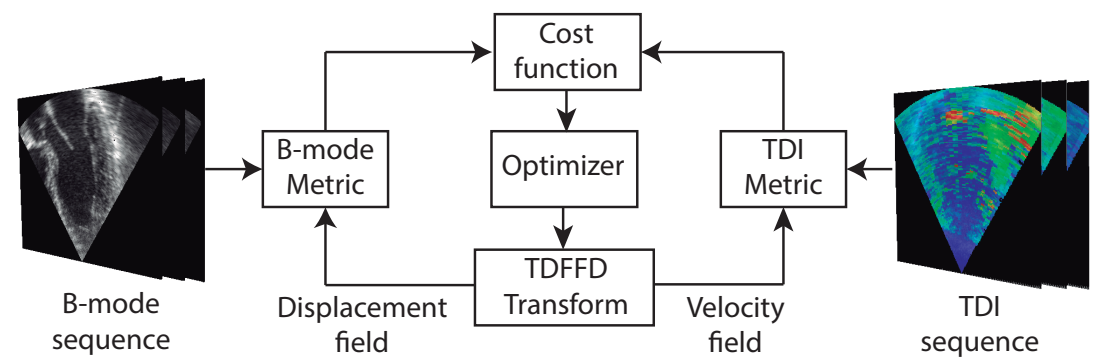

Fig. 1. Registration scheme. The optimizer finds the optimal parameters of the TDFFD transform by combining a voxel intensity based cost function and a metric that measures the similarity between the estimated velocities projected on the beam direction and the ones obtained from TDI.

$$
\left.\varphi_{0}(\mathbf{x}, T ; \mathbf{p})=\mathbf{x}+\int_{0}^{T} \mathbf{v}\left(\varphi_{0}(\mathbf{x}, t ; \mathbf{p}), t ; \mathbf{p}\right)\right) d t .
$$

Equation 2 can be discretized using a forward Euler integration scheme, converting the continuous $t$ in a sequence of discrete $t_{k}$. Thus, the transform at $t=t_{n}$ can be re-written as:

$$
\varphi_{0}\left(\mathbf{x}, t_{n} ; \mathbf{p}\right)=\mathbf{x}+\sum_{k=0}^{n-1} \mathbf{v}\left(\varphi_{0}\left(\mathbf{x}, t_{k} ; \mathbf{p}\right), t_{k} ; \mathbf{p}\right) \Delta t_{k}
$$

where $\Delta t_{k}=t_{k+1}-t_{k}$.

Note that the temporal sampling has to be small enough to get an accurate approximation of the trajectory with Eq. 3. In this work, we are using images from two modalities with different temporal resolution. To exploit the higher temporal resolution of TDI, the initial temporal sampling of the transform was set to the average temporal spacing between TDI frames. As proposed in [7], to ensure invertibility, the Jacobian of the transform with respect the spatial coordinates is evaluated at every sampled point. If a point with non-positive determinant of the Jacobian is detected, the time step is reduced by a factor of 2 until a positive determinant is found (which is a neccessary condition for invertibility).

\subsection{Cost function}

Unlike the case of B-mode US image registration, not only image voxel intensities will be considered but also tissue velocities. Therefore, the cost function proposed will take into account two different terms, one for each information channel considered:

$$
C(\mathbf{p})=J(\mathbf{p})+\lambda D(\mathbf{p})
$$


where $J$ represents a similarity metric applied on the B-mode image sequence, $D$ is the similarity metric applied on the TDI data and $\lambda$ is a weight balancing the two terms. For the first term, a squared difference of pixel intensities between the reference frame and the remaining frames is used as proposed in [7]. For the second term, a comparison between the projection of the velocity field estimated on the beam direction and the Doppler velocities is proposed. Unlike in [7], no incompressibility term was used. Thus:

$$
\begin{aligned}
C(\mathbf{p})= & \sum_{n=0}^{N} \sum_{\mathbf{x} \in \Omega_{I}}\left(I_{n}\left(\varphi_{0}\left(\mathbf{x}, t_{n} ; \mathbf{p}\right)\right)-I_{0}(\mathbf{x})\right)^{2}+ \\
& \lambda \sum_{m=0}^{M} \sum_{\mathbf{x} \in \Omega_{V}}\left(\mathbf{b}\left(\mathbf{x}, t_{m}\right) \cdot \mathbf{v}\left(\varphi_{0}\left(\mathbf{x}, t_{m} ; \mathbf{p}\right), t_{m} ; \mathbf{p}\right)-V\left(\mathbf{x}, t_{m}\right)\right)^{2}
\end{aligned}
$$

where $I_{n}$ represents a B-mode US frame at $t=t_{n}, \mathbf{b}$ is a unitary vector in the beam direction, $\mathbf{v}$ represents the velocity estimated, $V$ is the velocity value provided by TDI, $N+1$ and $M+1$ are the number of B-mode and tissue Doppler frames, and $\Omega_{I}$ and $\Omega_{V}$ correspond to the spatial domain of the B-mode and tissue Doppler images respectively.

\subsection{Optimizer and metric derivation}

Since the number of transform parameters is high, a L-BFGS-B optimizer [8] was preferred for registration. This algorithm uses the derivative of the cost function with respect to the parameters to find the optimal value of $\mathbf{p}$. Optimizer bounds were set to $\pm 20 \mathrm{~cm} / \mathrm{s}$. The total derivative of the cost function is:

$$
\begin{array}{cc}
\frac{d C(\mathbf{p})}{d \mathbf{p}}= & \sum_{n=0}^{N} \sum_{\mathbf{x} \in \Omega_{I}} 2\left(I_{n}\left(\mathbf{x}_{0}^{n}\right)-I_{0}(\mathbf{x})\right) \frac{d \mathbf{x}_{0}^{n}}{d \mathbf{p}} \cdot \nabla I_{n}\left(\mathbf{x}_{0}^{n}\right)+ \\
& \lambda \sum_{m=0}^{M} \sum_{\mathbf{x} \in \Omega_{V}} 2\left(\mathbf{b}\left(\mathbf{x}, t_{m}\right) \cdot \mathbf{v}\left(\mathbf{x}_{0}^{m}, t_{m} ; \mathbf{p}\right)-V\left(\mathbf{x}, t_{m}\right)\right) \mathbf{b}\left(\mathbf{x}, t_{m}\right) \frac{d \mathbf{v}\left(\mathbf{x}_{0}^{m}, t_{m} ; \mathbf{p}\right)}{d \mathbf{p}}(6)
\end{array}
$$

where $\nabla I$ represents the gradient of the image. For simplicity, $\varphi_{0}\left(\mathbf{x}, t_{n} ; \mathbf{p}\right)$ was written as $\mathbf{x}_{0}^{n}$. The steps for the computation of $\frac{d \mathbf{x}_{0}^{n}}{d \mathbf{p}}$ can be found in [7]. Note that the calculation of $\frac{d \mathbf{v}}{d \mathbf{p}}$ corresponds to the derivative of the B-spline used to model the velocity field.

\section{Experiments and results}

To validate the proposed algorithm, synthetic B-mode images and TDI data were used. The method was also applied to one healthy volunteer and the results were compared with the ones obtained from widely used commercial software.

For all the experiments, the B-spline grid used to model the velocity field had a size of $8 \times 8 \times N$ control points, where $N$ is the number of frames in the B-mode image sequence. 


\subsection{Validation with synthetic data}

The US synthetic dataset created in the second Cardiac Motion Analysis Challenge at STACOM-MICCAI $2012{ }^{6}$ was used to evaluate the accuracy of the myocardial motion estimated with the proposed method. This dataset was generated using heart segmentations from magnetic resonance images as input for the Bestel-Clement-Sorine electro-mechanical model [9][10]. The model was then used to simulate acute ischemia and dyssynchrony. For each simulated case, ground truth for displacement at every point was available. A US model (COLE, [11]) was used to generate synthetic 3D US images from the output of the electro-mechanical model. The simulated images consisted of $107 \times 80$ lines in azimuth and elevation direction over an angle of $80 \times 80$ degrees, resulting in a frame rate of $30 \mathrm{~Hz}$ due to the use of parallel beam forming. After mapping to a Cartesian coordinate system, the final data sets consisted of $500 \times 400 \times 500$ isotropic voxels with a voxel size of $0.46 \mathrm{~mm}$. The dataset comprised eight different cases: two normal cases, four ischemic and two cases with dyssynchrony.

A 2D longitudinal plane was extracted from every 3D US image, simulating Bmode US images in a 4-chambers view. In addition, velocities were calculated from the ground truth meshes and projected on the beam direction to simulate TDI data. White noise was also added to the velocities [12], so that the signal-to-noise ratio was $12 \mathrm{~dB}$. Image regions without velocity information were set as random values below $1.6 \mathrm{~cm} / \mathrm{s}$ (which was used as Nyquist velocity).

The value of $\lambda$ (Eq. 4) was determined by heuristic testing on one of the normal cases. The average displacement error value during the whole cardiac cycle was calculated in the left ventricle for different values of $\lambda$. Results are reported in Fig. 2. Based on these results, a value of $\lambda=30$ was taken for all the experiments.

Fig. 3 shows the mean and standard deviation of the displacement error (considering both longitudinal and transversal components) along one cardiac cycle for each case when using only B-mode images and when using both B-mode images and TDI. As it can be observed, the error was reduced in all cases by using B-mode images together with tissue Doppler velocities.

\subsection{Application to clinical case}

Experiments with one healthy volunteer (age 40, male) were also performed and results were compared with commercial software that is widely used in the clinical environment (EchoPAC, GE Healthcare, Milwaukee, WI, USA). Longitudinal velocities and displacements were calculated separately from B-mode images and from TDI with this software at 4 points of the myocardium and compared with the ones estimated using our approach (see Fig. 5). These points were located at basal- and mid-levels of the left ventricle, for both septal and lateral walls, as illustrated in Fig. 4. Only points at basal- and mid-levels were analysed because the beam is roughly aligned with the longitudinal direction at these locations. The algorithm that EchoPAC uses for motion tracking from B-mode images is based on speckle tracking, while motion from TDI is computed by temporally integrating the recorded velocities. No drift correction was performed because it may hide the propagation of errors.

Displacement curves obtained with the different methods had similar and realistic shapes, although the maximum displacements presented variations depending on the method considered. The maximum displacement estimated by the commercial software

\footnotetext{
${ }^{6}$ http://www.physense.org/stacom2012/
} 


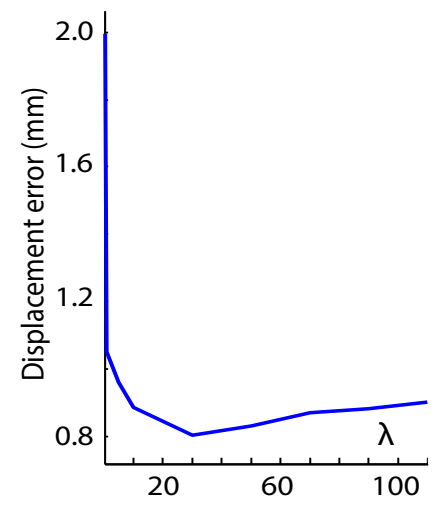

Fig. 2. Mean displacement error estimated during one cardiac cycle for different values of $\lambda$ in a normal case (Normal1).

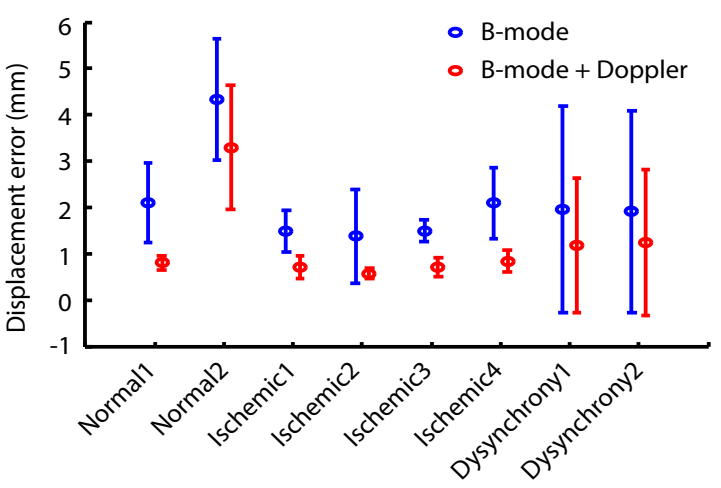

Fig. 3. Mean error (circles) and standard deviation (lines) calculated from registration results using only B-mode images (blue) and using both B-mode and TDI data (red) for every simulated case.

from B-mode images was higher in general. However, this increment in the magnitude could be due to the drift. In addition, if one region of the image has poor quality, tracking based only in B-mode sequences may fail, as it is the case of Point 1.

Displacements estimated with the commercial software from TDI and with our approach were very similar and presented a low drift compared to the results from Bmode images. However, since the displacement estimated from TDI data is calculated by temporally integrating the recorded velocities, the white noise that is present in the velocity signals may lead to errors in the estimation of the displacement. In addition, the limitation of using only TDI is that it only measures velocity on the beam direction. Our approach is more robust to errors by taking information from two sources.

The velocity curves estimated were very similar in shape and magnitude among the different approaches (with the exception of Point 1 for the estimation from B-mode images), while the velocity estimated with our approach is temporally smoother, this being due to its intrinsic definition through the TDFFD method.

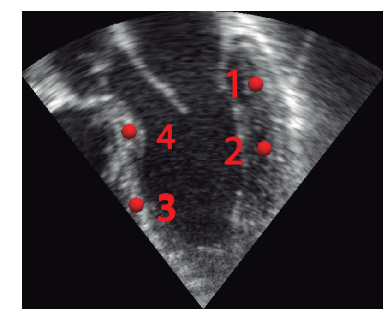

Fig. 4. Location of the points at which the comparison of Fig. 5 was performed. 


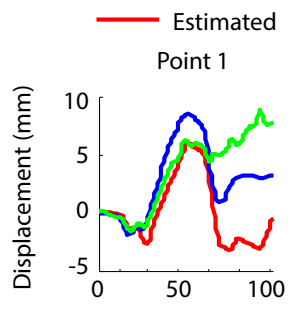

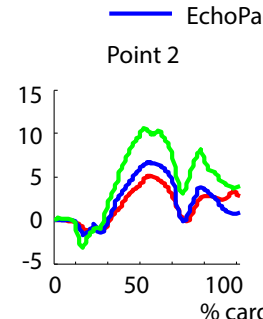

EchoPac Doppler

Point 3
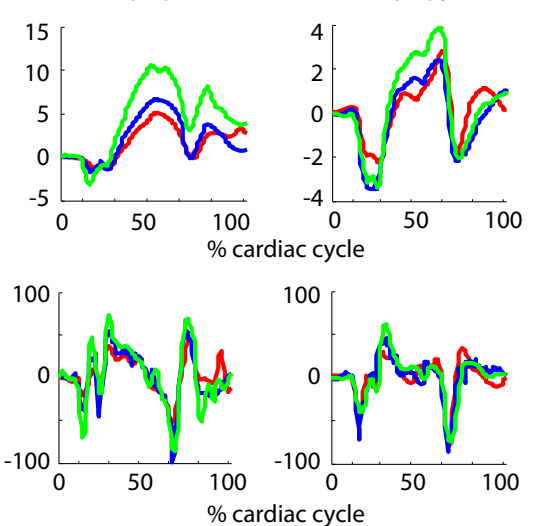

EchoPac B-mode

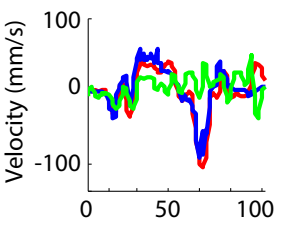

$\%$ cardiac cycle

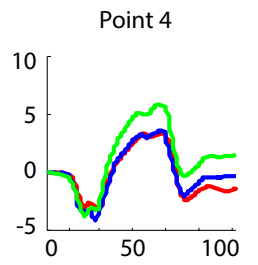

Fig. 5. Longitudinal displacements and velocities estimated during one cardiac cycle with: our approach (red), EchoPac using Doppler velocities (blue) and EchoPac using B-mode images (green).

\section{Conclusions}

We presented a registration framework with improved accuracy with respect to classical motion estimation from grayscale images, by combining B-mode echocardiographic sequences with the velocity information from tissue Doppler sequences. This approach allows increasing the temporal resolution of the estimated displacement field, while being independent from the beam angle. This could be very helpful to detect subtle differences in mechanics that are not visible when processing B-mode images or TDI separately. In addition, it is possible to control the influence of each information source by changing the value of the weighting parameter $\lambda$. Experiments on synthetic datasets demonstrated the higher accuracy reached by this approach. Moreover, experiments with one clinical case showed that the proposed method gave realistic results.

\section{References}

1. Szmigielski, C., Rajpoot, K., Grau, V., Myerson, S.G., Holloway, C., Noble, J.A., Kerber, R., Becher, H.: Real-time 3D fusion echocardiography. JACC Cardiovasc Imaging 3(7) (Jul 2010) 682-690

2. Rajpoot, K., Grau, V., Noble, J.A., Becher, H., Szmigielski, C.: The evaluation of single-view and multi-view fusion 3D echocardiography using image-driven segmentation and tracking. Med Image Anal 15(4) (Aug 2011) 514-528

3. Grau, V., Szmigielski, C., Becher, H., Noble, J.: Combining apical and parasternal views to improve motion estimation in real-time 3D echocardiographic sequences. In: Biomedical Imaging: From Nano to Macro. IEEE ISBI. (may 2008) 516 -519

4. Piella, G., De Craene, M., Butakoff, C., Grau, V., Yao, C., Nedjati-Gilani, S., Penney, G.P., Frangi, A.F.: Multiview diffeomorphic registration: Application to 
motion and strain estimation from 3D echocardiography. Med Image Anal (2013) http://www.sciencedirect.com/science/article/pii/S1361841513000030.

5. Amundsen, B.H., Crosby, J., Steen, P.A., Torp, H., Slrdahl, S.A., Stylen, A.: Regional myocardial long-axis strain and strain rate measured by different tissue doppler and speckle tracking echocardiography methods: a comparison with tagged magnetic resonance imaging. Eur J Echocardiogr 10(2) (2009) 229-237

6. Suhling, M., Arigovindan, M., Jansen, C., Hunziker, P., Unser, M.: Bimodal my-ocardial motion analysis from B-mode and tissue doppler ultrasound. In: Biomed-ical Imaging: From Nano to Macro. IEEE ISBI. (april 2004) 308 - 311

7. Del.Graene, M., Piella, G., Camara, O., Duchateau, N., Silva, E., Doltra, A., D'hooge, J., Brugada, J., Sitges, M., Frangi, A.F.: Temporal diffeomorphic free-form deformation: Application to motion and strain estimation from 3D echocardiography. Med Image Anal 16(2) (2012) 427 - 450

8. Byrd, R.H., Lu, P., Nocedal, J., Zhu, C.: A limited memory algorithm for bound constrained optimization. SIAM J. Sci. Comput. 16(5) (September 1995) 11901208

9. Chapelle, D., Le Tallec, P., Moireau, P., Sorine, M.: An energy-preserving muscle tissue model: formulation and compatible discretizations. International Journal for Multiscale Computational Engineering 10(2) (2012) 189-211

10. Marchesseau, S., Delingette, H., Sermesant, M., Ayache, N.: Fast parameter calibration of a cardiac electromechanical model from medical images based on the unscented transform. Biomech Model Mechanobiol (Oct 2012)

11. Gao, H., Choi, H.F., Claus, P., Boonen, S., Jaecques, S., Van Lenthe, G.H., Van der Perre, G., Lauriks, W., D'hooge, J.: A fast convolution-based methodology to simulate 2-d/3-d cardiac ultrasound images. IEEE Trans Ultrason Ferroelectr Freq Control 56(2) (Feb 2009) 404-409

12. Evans, D., Norman McDicken, W.: Doppler Ultrasound: Physics, Instrumentation and Signal Processing. Wiley; Second Edition (2000) 